\title{
42. Site of Origin of the Long-lasting Discharges in Frog Muscle Spindle
}

\author{
By Fumio Iто \\ Department of Physiology, School of Dentistry, \\ Aichi-Gakuin University, Nagoya \\ (Comm. by Teizo OgAwA, M. J. A., Feb. 12, 1970)
}

Discharges of muscle spindle receptors are affected by the polarization of the nerve ending $\mathrm{s}^{1)-3)}$ : The application of a direct current through a cathode near the receptor increased the frequency of firing, while the reversed current reduced the frequency or abolished the firing. ${ }^{2), 3)}$ It has been suggested that the adaptation of the firing occurs in the membrane process which converts generator currents into a sequence of action potentials. ${ }^{3)}$,4) In the present study, the exact location possessing an ability to produce the long-lasting discharges in the frog muscle spindle was studied.

Eighteen muscle spindles were isolated from the frog sartorius muscle. The procedure of the experiment and the paraffin gap method were essentially the same as described previously.5),6) The length of the paraffin gap along the parent axon was $2 \mathrm{~mm}$ in all the experiment while the distance from the capsule of a spindle receptor to the paraffin was changed. A bridge circuit was provided for direct current stimulation of the isolated nerve, as illustrated in Fig. 1A. All the experiments were carried out in the Ringer solution at $23-25^{\circ} \mathrm{C}$.

Fig. 1B shows spontaneous discharges of a spindle receptor and also firings in response to the application of a catelectrotonic and an anelectrotonic current to the nerve ending. The frequency of the spontaneous discharge was 5.2 impulses/sec on an average at a muscle length stretched by $2 \mathrm{~mm}$ from its in situ length. In an experimental condition where the paraffin gap was set less than $0.5 \mathrm{~mm}$ apart from the capsule, the application of a catelectrotonic current of $1.5 \times 10^{-7} \mathrm{~A}$ resulted in an appreciable facilitation of the spontaneous afferent discharge up to a mean frequency of 8.9 impulses/sec, while an anelectrotonic current of the same amount depressed the discharge (2.1 impulses/sec) and provoked simultaneously a burst of antidromic discharges, as illustrated in Fig. 1Ba.

The strength of the catelectrotonic current necessary for producing an appreciable facilitation of the afferent discharge increased with lengthening of the distance between the paraffin and the capsule; $2 \times 10^{-7} \mathrm{~A}$ for $1 \mathrm{~mm}, 2.5 \times 10^{-7} \mathrm{~A}$ for $2 \mathrm{~mm}$, and $5 \times 10^{-7} \mathrm{~A}$ for $5 \mathrm{~mm}$. 
A

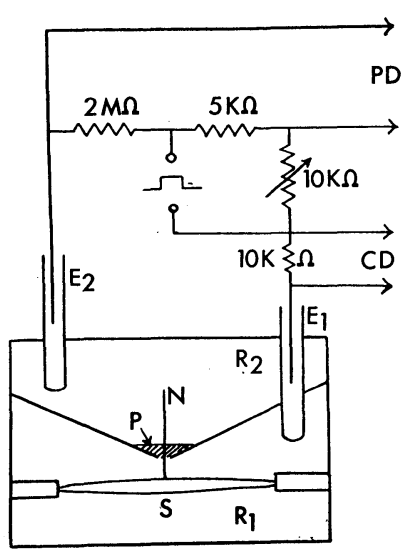

B

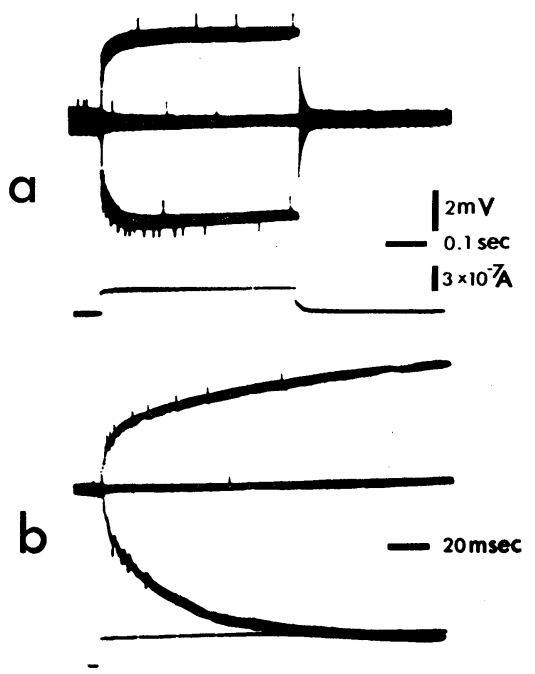

Fig. 1. A: Experimental arrangement. An isolated spindle receptor (S) enclosed by some muscle fibers was placed in a Ringer pool $\left(R_{1}\right)$ in a perspex box, and the nerve $(N)$ was passed into another Ringer pool $\left(R_{2}\right)$ through a liquid paraffin pool $(\mathrm{P})$. Responses of the receptor and the axon were led with a pair of calomel electrodes $\left(\mathrm{E}_{1,2}\right)$ and were displayed on a dual beam oscilloscope (PD) through a high input-impedance DC amplifier, in conjunction with a bridge circuit which was employed to pass through the calomel electrodes $\left(E_{1,2}\right)$ rectangular pulses of current with a duration of 0.5 sec. The current was detected as a potential drop across a $10 \mathrm{~K} \Omega$ resistor inserted into the bridge circuit (CD).

B: Records of action potentials from a muscle spindle receptor (upper traces) in the muscle stretched by $2 \mathrm{~mm}$ from its in situ length, without electrotonus (middle line) and during a catelectrotonus (upward deflection) and an anelectrotonus (downward deflection). The paraffin gap of $2 \mathrm{~mm}$ in length was situated within $0.5 \mathrm{~mm}$ from the spindle capsule (a) and at a distance $4 \mathrm{~mm}$ apart from it (b). The potentials recorded were due to current along the external longitudinal resistance (1-3 $\mathrm{M} \Omega$ ) of the axon in the paraffin pool. In all records negativity at the electrode $\mathrm{E}_{1}$ relative to the electrode $\mathrm{E}_{2}$ was displayed as an upward deflection. Upward deflection of the current records (lower traces) denotes the direction of the current flowing from electrode $\mathrm{E}_{2}$ to $\mathrm{E}_{1}$, and no record for the reversal current of the same amount was presented.

The depressing effect of the anelectrotonic current on the afferent discharge diminished also with increase in the distance between the paraffin and the capsule, but with the current of $4 \times 10^{-7}$ A or more the effect was preserved even at the proximal portion $10 \mathrm{~mm}$ apart from the capsule. With increase of the distance between the paraffin and the capsule, the threshold amount of the anelectrotonic current for eliciting the antidromic discharges also increased, and the discharge 
became to adapt rapidly. Fig. $1 \mathrm{Bb}$ shows responses during electrotonic current of $4 \times 10^{-7}$ A flowed through a paraffin gap situated at the axonal portion $4 \mathrm{~mm}$ apart from the capsule. During the catelectrotonus, no appreciable facilitation of the spontaneous afferent discharge was observed, but the initial burst of discharges appeared. The burst discharge adapted more rapidly than the discharge induced at the portion $2 \mathrm{~mm}$ apart from the capsule as demonstrated in Fig. $1 \mathrm{Ba}$ as the antidromic discharges. More rapidly adapting discharges were observed in the antidromic discharges in Fig. 1Bb which should initiate at the portion $6 \mathrm{~mm}$ apart from the capsule.

The above results suggest that a limited portion in the spindle nerve terminal possesses an ability to produce long-lasting discharges but the parent axon proximal more than $0.5 \mathrm{~mm}$ apart from the spindle capsule does not. This is in well agreement with the recent report of Nakajima and Onodera ${ }^{8)}$ that the slowly adapting stretch receptor in crayfish is able to elicit long-lasting repetitive discharges by a constant current stimulation to the axonal region near the soma. A supposition that the whole length of the parent axon, including the dorsal root, lacks the ability to produce sustained discharges is in a line with the preceding report ${ }^{9}$ that no long-lasting antidromic discharges into a spindle can be provoked by stimulation of the dorsal root filament. Ito ${ }^{10)}$ has recently demonstrated that a propagating impulse can be initiated transiently at a proximal portion along the extracapsular parent axon when temperature of the preparation is raised or the spindle receptor is stimulated strongly. It is quite conceivable from the present experiment that such a transient discharge adapts rapidly.

Acknowledgment. I am indebted to Dr. T. Satoh for his helpful discussions on this work. A part of this work was supported by a research grant from the Ministry of Education of Japan (97112).

\section{References}

1) Matthews, B. H. C.: J. Physiol., 71, 64-109 (1931).

2) Edwards, C.: J. Physiol., 127, 636-640 (1955).

3) Lippold, O. C. J., Nicholls, J. G., and Redfearn, J. W. T.: J. Physiol., 153, 209-217 (1960).

4) Emonet-Denand, F., and Houk, J.: Amer. J. Physiol., 216, 404-406 (1969).

5) Ito, F.: Proc. Japan Acad., 44, 852-855 (1968).

6) —: Jap. J. Physiol., 19, 373-391 (1969).

7) Grundfest, H.: Symposia on Quantitative Biology, 30, 1-14 (1965).

8) Nakajima, S., and Onodera, K.: J. Physiol., 200, 161-185 (1969).

9) Ito, F.: Jap. J. Physiol., 18, 731-745 (1968).

10) —: Jap. J. Physiol., 20 (1970) (to appear). 